\section{Boll. Accademia Gioenia di}

Scienze Naturali - Catania
BOLLAG Vol. 51, N. 381 (2018)

Full Paper, pp. FP1 - FP6

ISSN 0393-7143

doi:10.35352/gioenia.v51i381.1

Anno di fondazione 1824

\title{
Arithmetic Rings and their generalizations ${ }^{\dagger}$
}

\author{
Rosario Strano [1] * \\ [1] Dipartimento di Matematica e Informatica, Università di Catania
}

\begin{abstract}
Summary
Prüfer domains are characterized by various properties regarding ideals and operations between them. In this note we consider six of these properties. The natural generalization of the notion of Prüfer domain to the case of a commutative ring with unit, not necessarily a domain, is the notion of arithmetic ring. We ask if the previous properties characterize arithmetic ring in the case of a general commutative ring with unit. We prove that four of such properties characterize arithmetic rings while the remaining two are weaker and give rise to two different generalizations.
\end{abstract}

Key words: commutative rings, ideals, domains

\section{Riassunto}

I domini di Prüfer sono caratterizzati da varie proprietà riguardanti gli ideali ed operazioni fra essi. In questa nota consideriamo sei di tali proprietà. La naturale generalizzazione della nozione di dominio di Prüfer al caso di anelli commutativi con unità che non sono necessariamente domini è la nozione di anello aritmetico. Ci chiediamo allora se le precedenti proprietà caratterizzino gli anelli aritmetici nel caso generale di anelli commutativi con unità. Proviamo che quattro di tali proprietà caratterizzano gli anelli aritmetici mentre le rimanenti due sono più deboli e danno luogo a due diverse generalizzazioni.

Parole chiave: anelli commutativi, ideali, domini

\section{Introduction.}

Prüfer domains are characterized by various properties regarding ideals and operations between them. In particular we consider the following six properties for a domain $A$.

- 1) $(I+J): K=I: K+J: K$

for all $I, J, K$ ideals of $A$ with $K$ finitely generated

- 2) $I \cap(J+K)=I \cap J+I \cap K$

for all $I, J, K$ ideals of $A$

\footnotetext{
${ }^{\dagger}$ Paper presented by the member R. Strano in the session of March $2^{\text {st }}, 2018$
}

*e-mail: sstrano@dmi.unict.it 
- 3) $I: J+J: I=A$

for all $I, J$ finitely generated ideals of $A$

doi:10.35352/gioenia.v51i381.1

- 4) $I:(J \cap K)=I: J+I: K$

for all $I, J, K$ ideals of $A$ with $J, K$ finitely generated

- 5) $I \cdot(J \cap K)=I \cdot J \cap I \cdot K$

for all $I, J, K$ ideals of $A$

- 6) $(I \cap J) \cdot(I+J)=I \cdot J$

for all I, $J$ ideals of $A$.

They are all equivalent and characterize Prüfer domains among the commutative rings with unit wich are integral domains ( see [2] Th.25.2).

Now let $A$ be a commutative ring with unit, not necessarily an integral domain. we say that $A$ is an arithmetic ring if, for all maximal ideal $M$, the set of ideals of $A_{M}$ is linearly ordered by inclusion. This notion was introduced by J.P. Lafon in [1].

We ask if the arithmetic rings are characterized by the above properties among the commutative rings with unit.

In this paper we prove that the conditions 1), 2), 3), 4) are equivalent and characterize arithmetic rings. Moreover arithmetic rings satisfy condition 5) which in turn implies condition 6) (see Section 2).

The rings satisfying condition 6) have been studied and characterized in [4] and called quasiarithmetic rings. In Section 3 we recall the result of [4] and give a more explicit characterization.

In Section 4 we characterize rings satisfying condition 5) while in Section 5 we give an example of a ring satisfying 5) which is not arithmetic and an example of a ring satisfying 6) but not 5). Finally we consider the case of a noetherian ring.

All the proofs are elementary. All the rings we consider are commutative with unit; all the basic notions and properties about commutative rings can be found in [3].

\section{Arithmetic rings.}

First we recall the definition of arithmetic ring.

Definition 1 Let $A$ be a local ring and let $M$ be its maximal ideal; we say that $A$ is an arithmetic ring if it satisfies one of the equivalent conditions:

i) the set of ideals of $A$ is linearly ordered by inclusion,

ii) the set of principal ideals of $A$ is linearly ordered by inclusion,

iii) for all $x, y \in A$, the ideal $(x, y)$ is principal.

Let us prove the equivalence between i), ii) and iii). Clearly i) implies ii) and ii) implies iii).

Let us prove that iii) implies ii); put $(z)=(x, y)$ and assume $z \neq 0$ : we have $z=a x+b y, x=c z, y=$ $d z$; if one between $c$ or $d$ is invertible, e.g. $c$ invertible, we have $(z)=(x) \supseteq(y)$; otherwise we have $c, d \in M$ and $z=a c z+b d z$, i.e. $(1-a c-b d) z=0$; but $1-a c-b d$ is invertible which implies $z=0$.

Finally we prove that ii) implies i); let $I, J$ two ideals of $A$ and assume they are not comparable; hence there is $x \in I, x \notin J$ and $y \in J, y \notin I$; since $(x)$ and $(y)$ are comparable we assume $(x) \subseteq(y)$ hence $x \in J$.

Definition 2 Let $A$ be any ring; we say that $A$ is an arithmetic ring if $A_{M}$ is arithmetic, for any maximal ideal $M$.

Now we prove the following theorem. 
Theorem 1 Let A be a commutative ring with unit. Then the conditions 1), 2), 3), 4) are equivalent and characterize arithmetic rings.

doi:10.35352/gioenia.v51i381.1

Proof. The proof is the same as in the case of a domain ( see [2] Th.25.2). We recall it for the sake of completeness.

First we prove the equivalence between 1), 2), 3) and the condition:

(*) A is an arithmetic ring.

Since all conditions are local ( remember that $I: J$ localize if $J$ is finitely generated) we can assume that $A$ is a local ring.

If $A$ is an arithmetic local ring the set of ideals is linearly ordered by inclusion, hence it follows that the condition $(*)$ implies all the others.

Now we consider the following weaker condition of 3 ):

3') $(x):(y)+(y):(x)=A$ for all $x, y \in A$

and show that $\left.3^{\prime}\right)$ implies $(*)$.

In fact, il $A$ is a local ring with maximal ideal $M$, either $(x):(y)$ or $(y):(x)$ is not contained in $M$, hence either $(x):(y)=A$ or $(y):(x)=A$, i.e. either $(y) \subseteq(x)$ or $(x) \subseteq(y)$.

It remains to prove that 2) implies 3') and that 1) implies 3').

$\left.2) \Rightarrow 3^{\prime}\right)$. Let $x, y \in A$, we have

$$
(x) \subseteq(x) \cap((x+y)+(y))=(x) \cap(x+y)+(x) \cap(y)
$$

hence $x=\lambda+\mu$, where $\lambda=\rho(x+y)$ and $\mu=\sigma(y)$; then $x=\rho(x+y)+\sigma(y)$, i.e. $(1-\rho) x=(\sigma+\rho) y$; this implies $1-\rho=\tau \in(y):(x)$, moreover $\rho y=(1-\rho) x-\sigma y$ with $\sigma y=\mu \in(x)$, hence $\rho \in(x):(y) ;$ then $(x):(y)+(y):(x)=A$.

$1) \Rightarrow 3$ '). Let $x, y \in A$, we have

$$
A=((x)+(y)):((x)+(y))=(x):((x)+(y))+(y):((x)+(y))=(x):(y)+(y):(x) .
$$

Now we prove the equivalence between conditions 4$)$ and (*).

If $A$ is any ring and $I, J, K$ are ideals of $A$, we have $I:(J \cap K) \supseteq I: J+I: K$; for the other inclusion assume that $A$ is an arithmetic ring and let $M$ be any maximal ideal of $A$; we have

$$
(I:(J \cap K))_{M} \subseteq I_{M}:(J \cap K)_{M}=I_{M}:\left(J_{M} \cap K_{M}\right)
$$

Since $A$ is arithmetic we have $J_{M} \subseteq K_{M}$ or $K_{M} \subseteq J_{M}$; assume $J_{M} \subseteq K_{M}$ : we have

$$
I_{M}:\left(J_{M} \cap K_{M}\right)=I_{M}: J_{M}=I_{M}: J_{M}+I_{M}: K_{M}=(I: J)_{M}+(I: K)_{M}=(I: J+I: K)_{M}
$$

since $J, K$ are finitely generated.

Finally assume condition 4) and prove that $A$ is arithmetic or, equivalently, condition 3'). In fact, for any $x, y \in A$,

$$
A=((x) \cap(y)):((x) \cap(y))=((x) \cap(y)):(x)+((x) \cap(y)):(y)=(x):(y)+(y):(x) .
$$

The proof of theorem 1 is then complete.

It is easily seen, by localizing, that an arithmetic ring satisfies condition 5).

Finally we observe that condition 5) implies condition 6). In fact

$$
(I+J) \cdot(I \cap J)=(I+J) \cdot I \cap(I+J) \cdot J \supseteq I \cdot J
$$

and since the other inclusion is always true, the statement follows. 


\section{Quasi-arithmetic rings.}

In this section we study the rings satisfying condition 6). These rings were charadterizas and called quasi-arithmetic rings. Here we give a more explicit description. More precisely we prove the following theorem.

Theorem 2 Let A be a commutative ring with unit. Then the following conditions are equivalent: i) A satisfies condition 6) i.e.

$(I \cap J) \cdot(I+J)=I \cdot J$ for all $I, J$ ideals of A.

ii) For all maximal ideal $M$ of $A$ the ring $A_{M}$ satisfies the following condition:

for all $x, y \in A_{M}$ either $x y=0$ or the ideal $(x, y)$ is principal.

iii) For all maximal ideal $M$ of $A$ the ring $A_{M}$ satisfies the following condition:

for all $x, y \in A_{M}$ either $(x, y)^{2}=0$ or the ideal $(x, y)$ is principal.

$i v)$ For all maximal ideal $M$ of $A$ the ring $A_{M}$ satisfies the following condition:

the set $\mathcal{N}$ of elements of $A_{M}$ with square 0 form an ideal of $A_{M}$ with $\mathcal{N}^{2}=0$ which is comparable with any other ideal of $A_{M}$, and $A_{M} / \mathcal{N}$ is an arithmetic ring.

Rings satisfying the previous equivalent conditions are called quasi-arithmetic rings.

Proof of theorem 2. The equivalence between $i$ ), ii), iii) have been proved in [4]. Now we prove that $i i i) \Leftrightarrow i v)$.

iii) $\Rightarrow i v)$. Let $x, y \in \mathcal{N}$ and assume $x y \neq 0$, then $(x)$ and $(y)$ are comparable; suppose $x=\lambda y$, then $x y=\lambda y^{2}=0$, absurd. Then $x y=0$, hence $(x, y)^{2}=0$; this proves that $\mathcal{N}$ is an ideal and $\mathcal{N}^{2}=0$.

Let now $x \notin \mathcal{N}$; then $x^{2} \neq 0$ and $x$ is comparable with every $z \in \mathcal{N}$. If were $x=\mu z$ then would be $x^{2}=\mu^{2} z^{2}=0$, absurd; hence $z=\lambda_{z} x$ for all $z \in \mathcal{N}$ and then $\mathcal{N} \subset(x)$. We proved that every ideal of $A_{M}$ not contained in $\mathcal{N}$ contains it.

Finally let $\bar{x}, \bar{y}$ two non zero elements of $A_{M} / \mathcal{N}$; they are images of two elements $x, y \in A_{M}$ with $x^{2} \neq 0, y^{2} \neq 0$; then $(x),(y)$ are comparable and then also $\bar{x}, \bar{y}$ are comparable.

$i v) \Rightarrow$ iii). Let $x, y \in A_{M}$. If both are in $\mathcal{N}$ then since $\mathcal{N}^{2}=0$, is also $(x, y)^{2}=0$; if $x \notin \mathcal{N}, y \in \mathcal{N}$, the $(x) \supset \mathcal{N} \supseteq(y)$; if both $x, y$ are not in $\mathcal{N}$ then both contain $\mathcal{N}$ and since $A_{M} / \mathcal{N}$ is an arithmetic ring, $(x),(y)$ are comparable. The prof of theorem 2 is then complete.

\section{Rings satisfying condition 5).}

In this section we study the rings that satisfy condition 5). These rings fom a class intermediate between aritmetic and quasi-aritmetic rings. More precisely we prove the following theorem.

Theorem 3 Let $A$ be a commutative ring with unit. Then the following conditions are equivalent: i) A satisfies condition 5) i.e.

$I \cdot(J \cap K)=I \cdot J \cap I \cdot K$ for all $I, J, K$ ideals of $A$.

ii) For all maximal ideal $M$ of $A$ the ring $A_{M}$ satisfies the following condition:

for all $x, y \in A_{M}$ either $(x, y)^{2}=0$ and $\operatorname{ann}(x)=\operatorname{ann}(y)$ or the ideal $(x, y)$ is principal.

iii) $A$ is quasi-arithmetic and for all maximal ideal $M$ of $A$ the ring $A_{M}$ satisfies the following condition:

if $\mathcal{N}$ is the set of elements of $A_{M}$ with square 0 and $x, y$ are two non comparable elements in $\mathcal{N}$, then $\operatorname{ann}(x)=\operatorname{ann}(y)$.

Proof of theorem 3. ii) $\Rightarrow$ iii). From ii) follows that $A$ is quasi-arithmetic, moreover if $x, y \in \mathcal{N}$ are non comparable elements is $(x, y)^{2}=0$ since $\mathcal{N}^{2}=0$, hence $\operatorname{ann}(x)=\operatorname{ann}(y)$.

iii) $\Rightarrow$ ii). Since $A$ is quasi-arithmetic, for every maximal ideal $M$ of $A$ and for every two elements in $A_{M}$ is $(x, y)^{2}=0$ or the ideal $(x, y)$ is principal. Moreover if $(x, y)^{2}=0$ it is $x, y \in \mathcal{N}$ hence either the ideal $(x, y)$ is principal or $\operatorname{ann}(x)=\operatorname{ann}(y)$.

$i) \Rightarrow$ iii). Since $A$ satisfies 5) and 5) implies 6), $A$ is quasi-arithmetic. Moreover if $M$ is any maximal ideal of $A$ and $\mathcal{N}$ is the set of elements of $A_{M}$ with square 0 , let $x, y$ are two non comparable elements in $\mathcal{N}$. 
Assume that $\operatorname{ann}(x) \neq \operatorname{ann}(y)$ and assume that $t \in \operatorname{ann}(x)$ and $t \notin \operatorname{ann}(y)$, then $x t=0, y t \neq 0$. Hence

$$
t y=t(x+y) \in(t)(y) \cap(t)(x+y)=(t)((y) \cap(x+y)) \text { by } i) \quad \text { doi:10.35352/gioenia.v51i381.1 }
$$

hence $t y=t u$ with $u \in(y) \cap(x+y)$. It follows $u=\lambda y=\mu(x+y)$ and hence $t y=t u=\lambda t y$ i.e. $(1-\lambda) t y$ and since $t y \neq 0$ follows that $1-\lambda$ is in the maximal ideal $M A_{M}$ and hence $\lambda$ is invertible. In the same way $\mu$ is invertible hence $(u)=(y)=(x+y)$ and hence $(y, x+y)=(x, y)$ is a principal ideal.

iii) $\Rightarrow i$ ). Since $i$ ) is a local condition we can assume that $A$ is local with maximal ideal $M$. For $I, J, K$ ideals of $A$ we have to prove $I \cdot(J \cap K) \supseteq I \cdot J \cap I \cdot K$ since the other inclusion is always true. If the ideals $J, K$ are comparable the property is true, hence we can assume $J, K \subseteq \mathcal{N}$. If also $I \subseteq \mathcal{N}$ all product are zero hence the property is true. So we assume $I \supseteq \mathcal{N}$.

We observe first that every element of $I \cdot J$ is of the form $x y$ with $x \in I, y \in J$ : in fact let $z=\sum_{i=0}^{n} x_{i} y_{i}$ be an element of $I \cdot J$ with $x_{i} \in I, y_{i} \in J, i=1 \ldots n$; by induction we can suppose $n=2$ and $x_{1} y_{1}, x_{2} y_{2}$ different from zero; then $x_{1}, x_{2}$ are not in $\mathcal{N}$, hence are comparable; assume $x_{2}=\lambda x_{1}$ then $z=x_{1} y_{1}+\lambda x_{1} y_{2}=x_{1}\left(y_{1}+\lambda y_{2}\right)$.

Now let $u \neq 0$ be an element of $I \cdot J \cap I \cdot K$; we have $u=x y=x^{\prime} z$ with $x, x^{\prime} \in I, y \in J, z \in K$. Since $x, x^{\prime}$ are not in $\mathcal{N}$ ( otherwise $u=0$ ) they are comparable; assume $x^{\prime}=\mu x$; hence $u=x y=x z^{\prime}$ where $z^{\prime}=\mu z$ and $y, z^{\prime} \in \mathcal{N}, x \notin \mathcal{N}$.

Let us consider $y-z^{\prime}=t$; it is $x t=0$ but $x y \neq 0$, hence $x \in \operatorname{ann}(t), x \notin \operatorname{ann}(y)$ and by iii) the ideal $(t, y)=\left(y, z^{\prime}\right)$ is principal; hence $y$ and $z^{\prime}$ are comparable. Assume $z^{\prime}=\rho y$ : is $u=x z^{\prime}$ with $x \in I, z^{\prime} \in J \cap K$.

The prof of theorem 3 is then complete.

\section{$5 \quad$ Final observations.}

First we show that the three classes of rings we considered are all distinct. In fact we produce two examples: one of a ring satisfying condition 5) which is not arithmetic (Example 1) and one of a ring which is quasi-arithmetic and do not satisfies condition 5) (Example 2).

Example 1 Let $A$ be the ring which is the quotient of the domain

$$
k[[x]]+(y, z) k((x))[y, z]
$$

where $k$ is any field, with respect to the ideal $(y, z)^{2}$; we can write A as

$$
A=k[[x]]+y^{\prime} k((x))+z^{\prime} k((x))
$$

where $y^{\prime}, z^{\prime}$ are the canonical images of $y, z$ respectively, and where we have $y^{\prime 2}=y^{\prime} z^{\prime}=z^{\prime 2}=0$.

$A$ is a local ring whose maximal ideal is generated by $x$ and has a chain

$$
(x) \supset\left(x^{2}\right) \supset \cdots \supset \mathcal{N}=\left(y^{\prime}, z^{\prime}\right) .
$$

$A$ is not arithmetic since $y^{\prime}, z^{\prime}$ are not comparable, but it is easy to see that satisfies condition 5) since it is quasi-arithmetic and all non zero elements of $\mathcal{N}$ have annihilator equal to $\mathcal{N}$.

Example 2 Let $A^{\prime}$ be the quotient ring of the ring A considered in Example 1, with respect to the ideal $\left(x y^{\prime}\right)$.

$A^{\prime}$ is quasi-arithmetic but does not satisfy condition 5) since, denoting by $\bar{x}, \bar{y}, \bar{z}$ the canonical images of $x, y^{\prime}, z^{\prime}$ respectively, we have $\operatorname{ann}(\bar{y})=(\bar{x}, \bar{y}, \bar{z})$ while ann $(\bar{z})=(\bar{y}, \bar{z})$.

As a final remark we consider the case of a noetherian ring.

Remark Let $A$ be a noetherian ring. We have seen in [4] Proposition 2 that $A$ is quasi-arithmetic if and only if, for all maximal ideal $M$ of $A$ the local ring ring $A_{M}$, whose maximal ideal is $M A_{M}$, is one of the following types: 
a) a D.V.R. ( Discrete Valuation Ring),

b) an artinian ring with $M A_{M}$ principal,

c) an artinian ring with $\left(M A_{M}\right)^{2}=0$.

doi:10.35352/gioenia.v51i381.1

We observe that, in cases a), b), the local ring $A_{M}$ is arithmetic since all ideals are powers of the maximal ideal: this is well known in case a);

in case b) assuming that the maximal ideal is generated by (x), then, since $A_{M}$ is artinian, is $x^{r}=0$ for same positive integer $r$; now let $I \subseteq(x)$ be an ideal and let $t$ the least positive integer such that $x^{t} \in I$; it is easy to see that $I=\left(x^{t}\right)$.

In case c) the ring $A_{M}$ satisfies trivially condition 5).

In conclusion we can say that in the noetherian case the conditions 5) and 6) are equivalent.

\section{References}

[1] Lafon J.P. 1971, Anneax commutatifs sur lesquels tout module de type fini est somme direct de modules monogénes, J. of Algebra 17, 475

[2] Gilmer R. 1972, Multiplicative ideal theory, M. Dekker Inc, New York

[3] Atiyah M.F., Macdonald I.G. 1981, Introduzione all'algebra commutativa, Feltrinelli , Milano

[4] Strano R. 2014 , On quasi-arithmetic rings, Bollettino Accademia Gioenia 47, 377 